\title{
Questes
}

\section{Les corps de métier londoniens : artisans de la paix dans la cité?}

Laure Gevertz

\section{(2) OpenEdition}

Journals

\section{Édition électronique}

URL : http://journals.openedition.org/questes/192

DOI : $10.4000 /$ questes. 192

ISSN : 2109-9472

\section{Éditeur}

Les Amis de Questes

\section{Édition imprimée}

Date de publication : 25 septembre 2013

Pagination : 27-42

ISSN : 2102-7188

\section{Référence électronique}

Laure Gevertz, «Les corps de métier londoniens : artisans de la paix dans la cité ? », Questes [En ligne], 26 | 2013, mis en ligne le 01 janvier 2014, consulté le 20 avril 2019. URL : http:// journals.openedition.org/questes/192; DOI : 10.4000/questes.192 


\title{
Les corps de métier londoniens : artisans de la paix dans la cité ?
}

\author{
Laure GEVERTZ \\ Université Paris-Sorbonne (Paris IV)
}

L'influence exercée par les corps de métier londoniens sur l'économie urbaine au Moyen Âge a longtemps attiré l'attention des chercheurs et des historiens qui ont tenté, à travers l'étude des chartes et des ordonnances, de mesurer leur action sur le commerce et les échanges. Or loin de ne s'attacher qu'à réguler la production et la distribution des biens dans la cité, les associations professionnelles ont également joué un rôle non négligeable dans la gestion des rapports sociaux en participant notamment au maintien de la paix civile. Cette fonction apparaît très clairement dans les sources tout au long des $\mathrm{XIV}^{\mathrm{e}}$ et $\mathrm{XV}^{\mathrm{e}}$ siècles. Ainsi, le 3 octobre 1327, une proclamation visant à maintenir la paix du roi dans la cité interdit la constitution de toute forme de factions, ordonne à toute personne mécontente d'ester en justice ou de se plaindre auprès des dirigeants des métiers et charge ces derniers de maintenir leurs hommes au travail et de rapporter tout acte de rébellion aux autorités urbaines ${ }^{1}$. En 1417, le maire et les échevins londoniens, sur ordre du roi, commandent aux maîtres et gardiens des différents métiers de la cité de prendre des mesures pour empêcher les révoltes parmi les serviteurs et les apprentis et

\footnotetext{
${ }^{1}$ Arthur H. Thomas et Philip E. Jones, Calendar of Plea and Memoranda Rolls preserved among the archives of the Corporation of the city of London at the Guildhall, a. d. 1323-1364, London, Cambridge University Press, 1926, 2 volumes, vol. 1, p. 34.
} 
de leur rapporter les noms de tous les fauteurs de troubles ${ }^{2}$. Enfin, à la mort d'Édouard IV en 1483, les membres du Conseil du roi écrivent au maire de Londres pour qu'il convoque devant lui les gardiens de toutes les communautés d'artisans et de marchands de la ville aux côtés des responsables de la police urbaine et qu'il leur demande aide et assistance « contre toute personne cherchant à briser la paix du roi ou à lui porter atteinte $^{3} »$. Ces trois exemples montrent bien que les groupements professionnels sont considérés à l'époque comme des instruments efficaces au rétablissement de l'ordre, tant par la Couronne que par les autorités urbaines qui n'hésitent pas à faire appel à eux de manière récurrente. Ces exemples révèlent aussi le caractère ambigu de l'action judiciaire et policière des métiers en les présentant tantôt comme de simples auxiliaires tantôt comme de véritables substituts des pouvoirs urbains. Enfin, ils laissent supposer l'existence d'une juridiction spécifique exercée par les corps de métier sur leurs membres qui irait au-delà du seul encadrement professionnel et de la simple régulation des relations de travail.

Si les discours mettent en lumière, à travers des termes génériques, la participation des groupements professionnels à la préservation de la concorde entre les habitants de la cité, il convient de s'interroger sur la place exacte qu'ils occupent et sur la manière concrète dont ils interviennent dans les processus de résolution des conflits. On constate dans un premier temps que les corps de métier agissent à deux échelles : à celle de la cité, d'une part, puisqu'ils assistent ponctuellement les autorités urbaines dans des domaines qui relèvent de leurs compétences ou de leurs aptitudes particulières, et d'autre part à l'échelle de leur communauté

\footnotetext{
${ }^{2}$ Reginald R. Sharpe, Calendar of Letter-Books of preserver among the archives of the City of London at the Guildhall, Letter-BookI, a.d. 1400-1422, London, John E. Francis, 1909, p. 182.

${ }^{3}$ Laetitia Lyell et Frank D. Watney, Acts of Court of the Mercers' Company, 1453-1527, London, Cambridge University Press, 1936, p. 146-147.
} 
propre, où ils résolvent quotidiennement les disputes de toute nature susceptibles de survenir entre leurs membres. Dans un second temps, l'on peut observer que le travail de paix mis en œuvre par les groupements professionnels obéit à une série de procédés et de rituels cherchant à préserver l'idéal d'unité, d'amour et de solidarité qu'ils affichent.

\section{Les métiers, des agents de la paix urbaine?}

À la fin du Moyen Âge, les corps de métier londoniens semblent travailler activement à l'établissement de la paix à l'intérieur de la cité. Leur action pour restaurer l'ordre et la bonne entente entre les habitants s'exerce à la fois dans le domaine judiciaire et dans le domaine policier. Les représentants des associations professionnelles paraissent tout d'abord jouer un rôle de conseil auprès des autorités urbaines lors de règlements de querelles relevant de leurs compétences et de leurs savoirs particuliers. Le 25 avril 1355, par exemple, un cuisinier est accusé par l'un de ses clients devant le maire et les échevins d'avoir vendu du veau avarié. La viande incriminée est alors produite devant la cour qui décide de la soumettre à l'inspection de cinq autres cuisiniers. Ces derniers certifient indépendamment sous serment que la viande mise en cause est bonne et propre à être consommée et leur avis est suivi par les autorités urbaines qui acquittent l'accusé et déboutent le plaignant de son action ${ }^{4}$. Ce type de pratique, consistant à demander conseil auprès de professionnels afin d'orienter la décision judiciaire, s'observe dans des affaires extrêmement variées et ne se limite pas aux métiers de l'alimentation. Il tend même à devenir récurrent et à prendre une forme quasi institutionnelle dans certains domaines. Les procès en matière de biens immobiliers en offrent une parfaite illustration : quand ce genre d'affaires se présente, le maire et les

\footnotetext{
${ }^{4}$ Arthur H. Thomas et Philip E. Jones, Calendar of Plea and Memoranda Rolls preserved among the archives of the Corporation of the city of London at the Guildhall, a. d. 1323-1364, op. cit., p. 250.
} 
échevins semblent faire appel de manière systématique aux dirigeants de la compagnie des charpentiers et de la compagnie des maçons pour les aider dans leur prise de décision. Les archives urbaines montrent ainsi qu'entre 1437 et 1457 ces derniers interviennent dans chacun des huit litiges mettant en cause des propriétés foncières pour fournir des rapports détaillés destinés à servir de support aux autorités judiciaires et à les guider dans le prononcé de leur jugement ${ }^{5}$. Les gardiens des charpentiers et des maçons paraissent ainsi jouer un rôle d'experts judiciaires auprès du tribunal au service duquel ils mettent leurs compétences et leur légitimité reconnues en la matière. Ils se transforment dès lors en véritables auxiliaires officiels de la justice urbaine et ce d'autant plus qu'ils prêtent serment au maire et aux échevins avant leur entrée en charge.

À mesure que les associations professionnelles se structurent et accroissent leurs prérogatives et leurs privilèges par le biais de chartes et d'ordonnances, leurs dirigeants tendent progressivement à se substituer complètement dans le prononcé du droit et la résolution des disputes aux officiers de la cité dans les affaires qui nécessitent des connaissances techniques ou scientifiques très spécifiques. Les procès en matière de responsabilité médicale en témoignent : le 10 septembre 1424, le maître et le vice-maître des barbiers-chirurgiens, accompagnés de deux de leurs confrères, sont ainsi choisis comme arbitres, aux côtés d'un docteur et de deux bacheliers en médecine ainsi que d'un membre de la faculté de physique, pour juger s'il y a eu ou non erreur médicale lors du traitement d'une blessure à la main effectué par trois barbiers-chirurgiens sur un certain William Forest. Après avoir entendu les différents témoignages et examiné en détail la procédure de soin appliquée, les arbitres proclament

\footnotetext{
${ }^{5}$ Philip E. Jones, Calendar of Plea and Memoranda Rolls preserved among the archives of the Corporation of the city of London at the Guildhall, a.d.1437-1457, London, Cambridge University Press, 1954, vol. 5, p. 57, p. 69, p 75, p. 86, p. 96, p. 127, p. 151 et p. 156.
} 
que les accusés n'ont commis aucune faute et les absolvent de toutes les charges pesant contre eux ${ }^{6}$. En réunissant des représentants de la médecine pratique et de la médecine savante, la procédure arbitrale présente ici l'avantage d'intégrer dans la formation de jugement les personnes a priori les plus compétentes et les plus aptes à juger en la matière et à donner ainsi au litige une solution insusceptible de recours, sans avoir à faire intervenir les autorités urbaines.

Les associations professionnelles ne se contentent pas d'intervenir dans l'exercice de la justice, elles participent également au maintien de la paix dans la cité en fournissant une aide policière aux autorités urbaines. En août 1340, lorsqu'éclate un affrontement entre les poissonniers et les pelletiers, au cours duquel deux hommes trouvent la mort et d'autres sont gravement blessés, le maire ordonne aux responsables des deux métiers d'appréhender immédiatement les coupables qui ont réussi à prendre la fuite avant l'arrivée des officiers urbains et de les livrer aux sheriffs ${ }^{7}$. Ce procédé semble constituer un moyen efficace pour rétablir l'ordre et pour rendre rapidement la justice: de par l'existence des nombreux liens professionnels et personnels qui unissent entre eux les membres des associations corporatives, leurs représentants sont les mieux à même de connaitre l'identité des fautifs et de savoir où ces derniers sont susceptibles de se cacher. Ils ont, par conséquent, plus de chance de pouvoir les arrêter et de restaurer la paix dans les plus brefs délais.

C'est pourquoi le maire et les échevins décident de faire à nouveau appel aux dirigeants des deux métiers pour rétablir la paix quand un second conflit survient, trois ans plus tard, en novembre 1343. Pour restaurer la

\footnotetext{
${ }^{6}$ Arthur H. Thomas, Calendar of Plea and Memoranda Rolls preserved among the archives of the Corporation of the city of London at the Guildhall, a. d. 1413-1437, London, Cambridge University Press, 1943, vol. 4, p. 174.

${ }^{7}$ Arthur H. Thomas et Philip E. Jones, Calendar of Plea and Memoranda Rolls preserved among the archives of the Corporation of the city of London at the Guildhall, a. d. 1323-1364, London, Cambridge University Press, 1926, vol. 1, p. 126-127.
} 
paix, ils convoquent devant eux les principaux membres des poissonniers et des pelletiers, leur font prêter serment de dénoncer tous les membres de leur association soupçonnés d'avoir commis un méfait et leur donnent en outre le droit d'arrêter eux-mêmes ces derniers ${ }^{8}$. Les groupements professionnels se voient donc déléguer des pouvoirs en matière de police jusque-là exclusivement réservés aux officiers urbains : ils sont désormais directement inclus dans les dispositifs visant à maintenir la sûreté et la sécurité dans l'ensemble de la ville.

Les corps de métier représentent également un instrument utile pour rétablir l'ordre dans la cité même si leurs membres ne sont pas directement impliqués dans les conflits. En octobre 1483, des tondeurs de laine et des foulons, accompagnés d'une foule d'autres petits artisans, commencent à décharger la cargaison d'un navire en partance pour l'étranger, afin de protester contre les exportations de laine qui les privent de la matière première qu'ils travaillent et qui menacent ainsi leur emploi. Le maire et les échevins, ne parvenant pas à ramener les émeutiers à la raison, convoquent les membres de tous les groupements professionnels de la cité, sans exception - et pas seulement ceux des activités textiles concernées par la rébellion - pour qu'ils les accompagnent au port de la ville en armes et les aident à faire cesser les troubles. L'arrivée de cette force armée met en fuite les rebelles. Cependant, pour assurer un total retour à l'ordre, des gardes sont organisées conjointement par les officiers urbains et les corps de métier pour veiller à ce que les émeutes ne recommencent pas ${ }^{9}$. Cet événement illustre bien l'importance du soutien matériel que les métiers peuvent apporter aux autorités urbaines dans le maintien de la paix. Grâce

\footnotetext{
${ }^{8}$ Reginald R. Sharpe, Calendar of Letter-Books preserved among the archives of the city of London at the Guildhall, Letter-Book F, a.d.1337-1352, London, John E. Francis, 1906, p. 95-96.

${ }^{9}$ Laetitia Lyell et Frank D. Watney, Acts of Court of the Mercers' Company, 1453-1527, op. cit., p. 159-161.
} 
au grand nombre de personnes qui les composent et grâce à la force armée qu'ils peuvent représenter, mais également grâce à leur organisation structurée et hiérarchisée, les associations professionnelles peuvent compléter efficacement l'action des forces de police habituelles lorsque les effectifs limités de ces dernières se révèlent insuffisants pour rétablir l'ordre.

Cependant, si les corps de métier interviennent de manière ponctuelle, voire exceptionnelle, par l'usage de la force ou du droit, pour résoudre les conflits qui surviennent à l'échelle de la communauté urbaine, leur travail de paix s'exerce avant tout sur leurs propres membres, sur lesquels ils tendent à acquérir une véritable juridiction de droit commun.

\section{Trouver la paix au sein du métier}

Pour assurer leur pérennité et atteindre l'idéal d'amour et d'unité qu'elles n'ont de cesse d'afficher et de répéter, les associations professionnelles doivent travailler de manière constante à maintenir la paix et l'harmonie entre les personnes qui les composent. Cela est d'autant plus important que leurs membres s'inscrivent dans un réseau complexe de relations aussi bien professionnelles, amicales que familiales, ce qui peut susciter des concurrences et des tensions, sources potentielles de multiples conflits. C'est pourquoi les métiers, afin d'éviter d'exposer leurs querelles au regard extérieur et afin de préserver leur réputation et d'affermir leur légitimité, tentent de se doter progressivement de leur propre juridiction interne et d'obtenir le droit de régler tous les types de conflits susceptibles de survenir entre leurs membres et non plus seulement ceux ayant directement trait aux activités professionnelles qu'ils encadrent.

La compagnie des marchands de tissus offre un parfait exemple de cette évolution. En 1301, lors d'une dispute opposant un maître à son apprenti, le jugement est rendu par le maire et les échevins en présence 
d'autres confrères participant au procès à titre d'experts uniquement ${ }^{10}$. Dans cette affaire, un conflit interne est porté devant une instance extérieure, car la profession n'est pas encore suffisamment structurée et organisée pour disposer de ses propres instances juridictionnelles et trancher par elle-même le différend qui oppose ses deux membres. Or, l'article 4 des ordonnances promulguées le 20 juin 1347 par les «bonnes gens du métier pur unitee et bon amour entre eux norir» change radicalement la procédure à suivre en proclamant: «si nule debate, contracte ou querele de quel condicion q'il soyt sourde entre ascunes du dite mistere, qe nul face pleynte al leye tanqe il soyt moustré as mestres del mistere qe pur le temps serront et ent eyt responce ${ }^{11} »$. Désormais, les querelles internes doivent être résolues par les dirigeants du métier qui seuls peuvent décider de faire appel à la loi. Ces prescriptions sont strictement suivies, puisqu'en 1414 et en 1443 deux hommes sont punis pour avoir déposé des plaintes en justice sans en avoir obtenu l'autorisation préalable $^{12}$.

On assiste donc au milieu du XIII $^{\mathrm{e}}$ siècle à un phénomène de confiscation et de privatisation d'une partie de la justice par les corps de métier qui, de manière générale, tendent à interdire à leurs membres - ou tout du moins à leur restreindre fortement - les recours aux juridictions extérieures, qu'elles soient urbaines ou royales. Si les moyens et les mesures employés pour y parvenir diffèrent parfois, l'objectif visé semble bien identique: en faisant en sorte que tous les conflits internes soient examinés en première instance par les gardiens et en érigeant ces derniers en premier niveau de juridiction, les groupements professionnels évitent

\footnotetext{
${ }^{10}$ Reginald R. Sharpe, Calendar of Letter-Books preserved among the archives of the city of London at the Guildhall, Letter-Book C, London, John E. Francis, 1901, p. 188.

${ }^{11}$ Lisa Jefferson, The Medieval Account Books of the Mercers of London, Farnham, Ashgate, 2008, vol. 1, p. 44-49.

${ }^{12}$ Ibid., vol. 1, p. 271 et vol. 2, p. 653.
} 
d'afficher publiquement les disputes qui les agitent et renforcent ainsi l'autorité de leurs dirigeants et leurs moyens de contrôle afin d'assurer une parfaite cohésion entre leurs membres.

Les actes de la pratique témoignent clairement de l'élargissement des compétences judiciaires exercées par les corps de métier. Dès qu'ils en ont la capacité, ces derniers essayent de régler les disputes de toutes sortes qui surviennent quotidiennement en leur sein. Les registres des tailleurs montrent ainsi que leurs gardiens interviennent pour régler les conflits en matière de dettes qui jusque-là relevaient de la compétence des autorités urbaines. Le 26 novembre 1486, ils ordonnent à William Boston de payer la dette de 42 shillings 2 pence qu'il a contractée auprès d'un autre membre du métier, Roger Maynell, en un versement immédiat de 26 shilling 8 pence et de verser la somme restant due sous la forme de paiements trimestriels réguliers ${ }^{13}$. Le recours à une procédure judiciaire interne permet d'une part de régler rapidement le conflit sans faire intervenir d'acteurs extérieurs et d'autre part de chercher le compromis susceptible d'être le mieux accepté par les protagonistes.

Les corps de métier s'inscrivent ainsi dans un processus global de pacification des relations unissant les personnes qui les composent. Leurs dirigeants tranchent désormais les querelles ayant donné lieu à des agressions physiques ou verbales et se chargent de faire appliquer les législations existant en la matière. Suite à la proclamation royale de 1334 interdisant de brandir une épée, un couteau ou toute autre arme à l'intérieur de la cité, les groupements professionnels se mettent ainsi à juger euxmêmes ce type d'infractions sans en référer aux autorités urbaines ni à la

${ }^{13}$ Matthew Davies, The Merchant Taylor's Company of London: Court Minutes, 1486-1493, Stamford, Richard III and Yorkist History Trust, 2000, p. 82. 
Couronne $^{14}$. En 1352, l'orfèvre Petrus le Mazere est condamné par ses gardiens à une livre d'amende « pur son cotel treré a Johan Herwy et sanc gouté ${ }^{15} \gg$. Les corps de métier s'impliquent donc directement dans le maintien de l'ordre et dans la lutte contre les violences à l'échelle de leur communauté en participant au fonctionnement de la justice pénale. Ils n'hésitent pas à étendre leur travail de paix à toutes les sphères, y compris celle de la vie privée, et à intervenir dans le système des relations familiales et amicales. Le tailleur William Barton est ainsi jugé par les gardiens de son métier en 1491 pour avoir injurié l'un de ses confrères et l'épouse de celui-ci, sous l'effet d'une « rancœur brûlante » et « d'une grande colère » : il est condamné à leur demander publiquement pardon et à se rendre à leur domicile pour présenter ses excuses à l'épouse offensée ${ }^{16}$. Ces pratiques visent à pacifier les rapports qui unissent les membres du groupe professionnel en interdisant les paroles et les actes discourtois susceptibles d'engendrer des disputes. Elles cherchent à préserver la concorde et l'harmonie au sein de la communauté en veillant au respect de l'honneur de chacun. Le 8 avril 1487, trois tailleurs, Richard West, Stephane Jenyns et Thomas Randyll, sont par exemple contraints de promettre sous serment en leur nom et en celui de leur maisonnée de se comporter honnêtement envers leur confrère, Radulf Bukberd, et sa famille «tant en mots qu'en faits $^{17} \gg$.

Si les associations professionnelles élargissent progressivement leurs prérogatives judiciaires afin d'instaurer, et surtout de restaurer, l'amitié et la bonne entente indispensables à leur bon fonctionnement et à leur survie,

\footnotetext{
${ }^{14}$ Henry T. Riley, Memorials of London and London Life in the XIII ${ }^{h}, X I V^{t^{h}}$, and $X V^{\text {th }}$ Centuries: Being a Series of Extracts, Local, Social, and Political, from the Early Archives of the City of London, a. d. 1276-1419, London, Longmans, 1868, p. 42.

${ }^{15}$ Lisa Jefferson, Warden's Accounts and Court Minute Books of the Goldsmiths' Mistery of London, 1334-1446, Woodbridge, Boydell Press, 2003, p. 40.

${ }^{16}$ Matthew Davies, The Merchant Taylor's Company of London: Court Minutes, 1486-1493, op. cit., p. 217.

${ }^{17}$ Ibid., p. 131.
} 
leurs actions obéissent pour ce faire à des procédés et des rituels de paix extrêmement variés et codifiés.

\section{Procédés et rituels de paix}

Parmi les modes de résolution de conflit mis en œuvre par les corps de métier l'on distingue deux types de procédés différents. Le premier consiste à recourir au jugement des dirigeants du métier, assistés au besoin d'une cour plus large composée des personnes les plus illustres de la communauté, voire de l'assemblée entière des membres dans les affaires les plus graves. Les dirigeants du métier possèdent une autorité et une légitimité au sein du groupe qui leur permettent de conférer à leur jugement une vraie force exécutoire. Ils disposent qui plus est d'importants moyens de pression et de sanction pour se faire obéir : ils peuvent en effet exiger le paiement d'une amende, exclure quelqu'un de la fraternité ou encore l'envoyer en prison avec l'assistance des officiers urbains.

Le recours à l'arbitrage constitue le second procédé utilisé pour mettre un terme aux querelles et restaurer la concorde au sein des associations professionnelles. Ce mode d'action est fréquemment employé par les tailleurs : on dénombre dans leurs archives 91 procédures arbitrales entre 1486 et 1493 , ce qui fait une moyenne d'environ onze par $a{ }^{18}$. En règle générale, les dirigeants du métier désignent deux arbitres - mais ce nombre peut varier selon la complexité de l'affaire - et fixent une date limite à laquelle le verdict doit être rendu. Lorsque Henry Brewster et John Harreson sont choisis le 21 avril 1486 pour arbitrer une dispute, ils se voient ainsi fixer le 21 mai comme date butoir, ce qui leur donne un délai d'un mois pour élaborer une solution ${ }^{19}$. Les mentions présentes dans les registres insistent sur la neutralité des arbitres et sur l'attitude impartiale

${ }^{18}$ Matthew Davies, The Merchant Taylor's Company of London : Court Minutes, 1486-1493, op. cit.

${ }^{19}$ Ibid., p. 52. 
qu'ils doivent adopter pour régler le litige. Ces deux critères sont fondamentaux car ils conditionnent la reconnaissance par les parties de l'autorité et de la légitimité de ceux qui vont les juger: en acceptant librement les arbitres, elles s'engagent implicitement à respecter le verdict à venir ce qui marque déjà un pas vers l'apaisement. Le jugement unilatéral et l'arbitrage apparaissent comme deux procédures complémentaires, la première pouvant se substituer à la seconde en cas d'échec.

Quelle que soit la méthode employée pour résoudre le conflit, l'on constate cependant que les solutions adoptées visent moins à punir et à sanctionner qu'à rétablir l'harmonie et l'unité au sein du métier, comme en témoigne la mise en œuvre de gestes et de rituels de paix. Les sentences mettent en effet davantage l'accent sur la réconciliation et le pardon que sur le châtiment des fautifs. Les jugements cherchent avant tout à restaurer la concorde et l'amitié qui ont été brisées par des paroles ou des actes hostiles, ce qui explique qu'on puisse avoir, pour une même faute commise, deux peines différentes. En 1400, les dirigeants des marchands de tissus jugent successivement deux hommes accusés d'avoir menacé un confrère à l'aide d'un couteau. L'un est condamné à une amende de 40 shillings, mais, comme il accepte de se soumettre «bonement al ordenance del mistere », on lui pardonne et il n'a plus qu'à payer une somme modique de 6 shillings 8 pence $^{20}$. L'autre, au contraire, refuse d'accepter le jugement des gardiens du métier qui se voient contraints d'en appeler au maire et à la force publique. Après un séjour en prison, le contrevenant accepte finalement d'obéir à leur décision, il est alors libéré mais doit payer la totalité des 40 shillings d'amende ${ }^{21}$. Ces deux affaires montrent bien que ce qui est considéré comme le plus grave n'est pas tant de porter

${ }^{20}$ Lisa Jefferson, The Medieval Account Books of the Mercers of London, op. cit., vol. 1, p. 156.

${ }^{21}$ Ibid., p. 155156. 
ponctuellement atteinte à la paix en agressant quelqu'un physiquement que de remettre en question l'ordre et l'autorité établis en refusant d'obéir et de reconnaître son erreur. L'acceptation du jugement et la soumission aux autorités légitimes constituent en soi une étape importante du processus de pacification et de l'obtention du pardon.

Par ailleurs, on assiste, lors du prononcé des peines, à la mise en place d'une véritable " économie de l'oubli », pour reprendre l'expression utilisée de Nicolas Offenstadt ${ }^{22}$ : les jugements cherchent à abolir tout motif passé, présent ou futur, susceptible de mettre en péril la réconciliation. Ils organisent d'abord sur le plan matériel des procédures de restitution des biens et de compensation des pertes afin d'effacer les traces concrètes du conflit et de faire en sorte que personne ne soit lésé. Ainsi, faute de trouver une solution viable pour que l'orfèvre John Sutton et son apprenti puissent vivre et travailler ensemble paisiblement, les gardiens du métier décident de mettre un terme au contrat d'apprentissage qui les unit et de faire payer par l'apprenti à son employeur un dédommagement de 8 marcs : de cette façon, chacun reçoit une compensation, l'un recouvre son investissement et l'autre sa liberté ${ }^{23}$.

Le travail de paix implique également un effort des individus sur eux-mêmes, qui doit se traduire par l'effacement des rancœurs et des haines passées. Les juges tentent d'intervenir sur la mémoire des parties en les enjoignant à l'oubli et en leur imposant un silence perpétuel. Ce procédé se manifeste clairement dans un jugement rendu par les dirigeants des orfèvres le 18 juillet 1439 : ceux-ci déclarent mettre un terme à tout litige éventuel né « depuis le commencement du monde jusqu'à ce 18 juillet » et interdisent aux deux protagonistes de prononcer des paroles ou de

\footnotetext{
${ }^{22}$ Nicolas Offenstadt, Faire la paix au Moyen Âge, Paris, Olivier Jacob, 2007, p. 50.

${ }^{23}$ Lisa Jefferson, Warden's Accounts and Court Minute Books of the Goldsmiths' Mistery of London, 1334-1446, op. cit., p. 487.
} 
commettre des actes susceptibles de raviver la querelle ${ }^{24}$. Pour garantir la pérennité de la paix, les jugements recourent aussi à des procédés proches de l'« asseurement » employé au haut Moyen Âge. Les parties peuvent être soit contraintes de prêter oralement serment de respecter les termes de la réconciliation, soit de signer des peace bonds ou promesse de paix écrites. Ces deux pratiques présentent toutes deux un caractère préventif et incitatif dans la mesure où elles cherchent à éviter toute récidive du conflit en obligeant les parties à engager publiquement leur foi et leur honneur. Le 6 août 1491, les tailleurs Lyonelle Baker et John Syokall établissent ainsi mutuellement, en signe «de paix perpétuelle et d'amitié », une quittance générale pour tous leurs sujets de dispute et font une promesse de bien se comporter l'un envers l'autre en paroles comme en actes; ils seront condamnés à payer dans le futur une amende de $10 £$ s'ils n'honorent pas la parole donnée ${ }^{25}$.

Enfin l'on observe, pour pérenniser l'apaisement, la mise en œuvre de rituels visant à officialiser et à parfaire la réconciliation des parties en conflit et à offrir une reconnaissance objective de la paix. Le partage de nourriture opéré publiquement en constitue un parfait exemple: il symbolise l'unité et la bonne entente retrouvées et scelle concrètement le retour de l'amitié. La commensalité fonctionne comme un rite de paix et marque matériellement la réconciliation tout en sollicitant la mémoire des personnes qui ont assisté à la scène. C'est pourquoi, afin de clore la dispute qui a opposé William Porter à Robert Langford et Harry Pool, les gardiens des orfèvres leur ordonnent pour « la bonne fin de paix et l'amour qu'il doit $\mathrm{y}$ avoir entre $\operatorname{eux}^{26} \gg$ de s'offrir réciproquement un dîner en leur présence.

\footnotetext{
${ }^{24}$ Ibid., p. 498.

${ }^{25}$ Matthew Davies, The Merchant Taylor's Company of London: Court Minutes, 1486-1493, op. cit., p. 201.

${ }^{26}$ Lisa Jefferson, Warden's Accounts and Court Minute Books of the Goldsmiths' Mistery of London, 1334-1446, op. cit., p. 538.
} 
En l'espèce, le repas, prétexte au don et au contre-don de nourriture, permet de rétablir symboliquement la solidarité et l'entraide rompues par la dispute. L'échange de baiser et la poignée de main, employés eux aussi dans les rituels, procèdent d'une logique similaire mais apportent, grâce au toucher, une autre dimension. Ainsi, le $1^{\text {er }}$ février 1420 , les orfèvres William Shibourne et John Carlere sont enjoints par jugement d'embrasser à trois reprises "pour rapporter une paix complète » les deux hommes qu'ils ont offensés et, de la même manière, le 14 juin 1457, les marchands de tissus Symon Dawdley et Thomas Shelley se voient ordonner «de se prendre par la main et d'être amis ${ }^{27} »$. La poignée de main apparaît comme le signe de paix par excellence et matérialise, aux yeux de tous, l'amitié retrouvée entre les parties tandis que le baiser et l'accolade sont davantage des signes de pardon qui traduisent par le contact physique des corps la réconciliation des âmes. Comme le souligne bien Nicolas Offenstadt, la paix conclue par le contact des corps, "geste central de la paix publique » constitue un véritable «signe de basculement» et donne au rituel de pacification une pleine efficacité ${ }^{28}$.

Par le rôle important qu'ils jouent dans l'exercice de la justice et de la police au sein de la cité et par les procédés et les rituels de réconciliation qu'ils mettent en œuvre, les corps de métier londoniens participent donc de manière déterminante au maintien de la paix civile. Ils disposent pour résoudre les conflits d'un large éventail de procédés et de sanctions qui apporte souplesse, efficacité et rapidité à leur action. Cependant, si celle-ci s'observe à l'échelle de l'ensemble de la société urbaine, elle se concentre essentiellement sur les membres du groupe professionnel directement soumis à l'autorité corporative : les associations de métier cherchent avant

${ }^{27}$ Laetitia Lyell et Frank D. Watney, Acts of Court of the Mercers' Company, 1453-1527, op. cit., p. 46-47.

${ }^{28}$ Nicolas Offenstadt, Faire la paix au Moyen Âge, op. cit., p. 206. 
tout à préserver l'unité et la solidarité indispensables à leur bon fonctionnement et à leur pérennité. En outre, leur travail de paix s'inscrit dans une stratégie plus large qui vise à renforcer leur pouvoir et leur souveraineté : il s'insère dans le système des relations interpersonnelles en participant à son réordonnancement et à sa redéfinition. 